\title{
Endoscopically placed fiducials facilitate minimally invasive, parenchymal preserving resection of small pancreatic neuroendocrine tumors
}

\author{
Mohan K. Mallipeddi ${ }^{*}$, MD, Kevin N. Shah ${ }^{1}$, MD, Sabino Zani ${ }^{1}$, Rebecca A. Burbridge ${ }^{2}$, Rebekah R. White ${ }^{1}$, Dan G. Blazer ${ }^{1}$, Theodore N. \\ Pappas $^{1}$ and Alexander Perez ${ }^{1}$ \\ ${ }^{1}$ Department of Surgery, Duke University Health System, Durham, NC USA \\ ${ }^{2}$ Division of Gastroenterology - Department of Medicine, Duke University Health System, Durham, NC USA
}

\begin{abstract}
Introduction: Complete excision of early stage pancreatic neuroendocrine tumors (PNETs) is curative. However, small PNETs may be difficult to localize intraoperatively, leading to larger pancreatic resections than required. Prior reports suggest that preoperative endoscopic ultrasound guided placement of markers (fiducials) (EUS-F) facilitates tumor identification during open surgery. We describe our experience with this approach during minimally invasive surgery to preserve pancreatic parenchyma.
\end{abstract}

Methods: This is a retrospective case series of 3 patients since 2010 who underwent EUS-F followed by laparoscopic or robotic pancreatic resection of PNETs $\leq 2.5$ $\mathrm{cm}$. All patients underwent preoperative EUS-F by our gastroenterology service. We report the visibility of fiducials at surgery by ultrasound and/or fluoroscopy as well as their impact on the extent of surgery.

Results: Patients received 1 to 3 markers without complication. Final pathology revealed complete excision of a $25 \mathrm{~mm}$ nonfunctional uncinate tumor, 22 mm tail insulinoma, and $2.5 \mathrm{~mm}$ nonfunctional tail tumor. The uncinate tumor was identified using intraoperative ultrasound and enucleated. The two tail tumors were identified using fluoroscopy after failed attempts with ultrasound; these two patients underwent limited distal pancreatectomies. There was one self-limited pancreatic fistula.

Conclusions: Endoscopically placed fiducials facilitate reliable intra-operative identification of small PNETs allowing for minimally invasive parenchymal preserving surgery, such as enucleation or limited distal pancreatectomy. Fluoroscopy was the most reliable method of identifying the fiducials.

\section{Introduction}

Pancreatic neuroendocrine tumors (PNETs) are rare, affecting 1 per 500,000 people, and may cause an increased production of hormones such as insulin, gastrin, glucagon, vasoactive intestinal peptide, and somatostatin [1]. PNETs are becoming more frequently diagnosed, with a $710 \%$ increase over the last 22 years, mostly due to the widespread use of high resolution imaging $[2,3]$.

PNETs are staged according to size, lymph node involvement, and presence of distant metastases [4]. PNETs are graded histologically according to their degree of differentiation [4]. Early stage and low grade PNETs have the most favorable prognosis- $100 \% 5$ year survival rate for stage I compared to $55 \%$ for stage IV. ${ }^{5}$ Complete surgical resection is curative for these tumors [6,7]. For all stages and grades of PNETs, survival is better in those who undergo surgical resection [8].

Small PNETS $(<15 \mathrm{~mm})$ have very little risk of recurrence, lymph node involvement, or distant metastases [9-12]. These tumors should be resected with a parenchymal-preserving technique (enucleation) since they would not benefit from more extensive resections such as pancreaticoduodenectomy or distal pancreatectomy. While enucleation does results in a higher fistula rate $(21 \%)$ requiring prolonged drainage compared to pancreaticoduodenectomy, it also results in fistulas of less severity (International Study Group Pancreatic
Fistula Type A), less operative blood loss, less operative time, shorter length of intensive care unit stay, and shorter length of overall hospital stay [13-15]. Pancreaticoduodenectomy also results in new onset exocrine $(1.5 \%)$ and endocrine (4\%) insufficiencies that are not associated with enucleation [16-19]. Enucleation has been accepted as a standard surgical approach for these tumors since 1990 [20]. Most PNETs (56\%) are located in the pancreatic head/uncinate region [21]. Tumors of this area that are oncologically amenable to enucleation should proceed as such in order to avoid the complications associated with pancreaticoduodenectomy $[15,19]$.

Minimally invasive pancreatic surgery and pancreatic parenchymalsparing surgery for PNETs has become more popular and have proven to be feasible, safe, and reduce length of hospital stay, without increasing morbidity or compromising survival compared to its open counterpart [22-30]. Small, deep PNETs in the pancreatic uncinate may be difficult to localize intra-operatively and may explain why PNETs of the pancreatic head are less often resected and technically

Correspondence to: Dr. Mohan Mallipeddi, Department of Surgery, Duke University, Durham, USA; E-mail: mohan.mallipeddi@duke.edu

Key words: enucleation, fiducial, pancreatic neuroendocrine tumor

Received: April 14, 2016; Accepted: May 06, 2016; Published: May 11, 2016 
more challenging to enucleate laparoscopically [31].

Preoperative localization of pancreatic neuroendocrine tumors with traditional axial imaging fails in $40-60 \%$ of patients. Endoscopic ultrasound (EUS) is highly sensitive (93\%) in the detection of these PNETs [21]. Intra-operative localization with laparoscopic ultrasound has been shown to reduce the need for conversion to an open procedure to identify and guide resection of these tumors [30,32-35]. X-ray and echo-opaque markers (fiducials) are added to guide stereotactic body radiation therapy and lessening collateral damage [36]. These markers have been delivered via EUS for mediastinal and abdominal tumors and more recently applied safely, effectively, and specifically to the pancreas ( $2 \%$ complications, $7 \%$ migration, and $90 \%$ successful placement). These markers have aided open enucleation of pancreatic uncinate PNETs [37-40].

The aim of this report is to address the intra-operative localization facilitated by endoscopic ultrasound placed fiducials and the operative technique of laparoscopic enucleation of the PNET of the uncinate.

\section{Case report}

A 56 year old gentleman with a history of benign prostatic hypertrophy presented with hematuria. He was incidentally diagnosed with a $10 \mathrm{~mm}$ tumor in the pancreatic uncinate on computed tomography (Figure 1) without any symptoms or other concerning radiographic findings. He underwent further imaging with endoscopic ultrasound, which documented a well circumscribed oval-shaped 10 x $12 \mathrm{~mm}$ tumor without any suspicious appearing regional lymph nodes. A trans-duodenal EUS-guided biopsy of this tumor revealed a neuroendocrine tumor with cells testing positive for CD56, synaptophysin, chromogranin, and pan cytokeratin. Because of its low stage, laparoscopic enucleation was planned. Due to its small size and remote location in the uncinate, $3 \mathrm{x}$-ray and echo-opaque markers (Visicoil fiducials, Core Oncology, Santa Barbara, CA, USA) were placed in and around the tumor the day prior to surgery via a 22-gauge Cook Echo Tip ${ }^{\circledR}$ needle under EUS-guidance (Figure 2). No complications were associated with the placement of the fiducials, and no evidence of pancreatitis was shown either clinically or on surgical pathology. Final pathology confirmed an early stage (1B, 2.5 x 2.0 x 2.0 $\mathrm{cm}$ ) and low grade (moderate differentiation) neuroendocrine tumor confined to pancreas with 1 mitosis per 2 square $\mathrm{mm}$, without tumor necrosis or lymphatic-vascular or perineural invasion and image cytometry of Ki-67 labeling index of proliferation of $9 \%$.

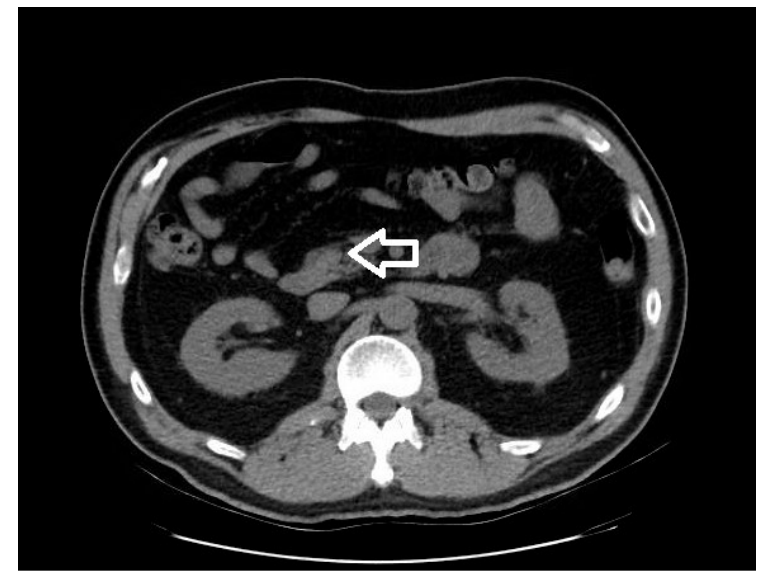

Figure 1. Computed tomography demonstrating uncinate tumor (white arrow).

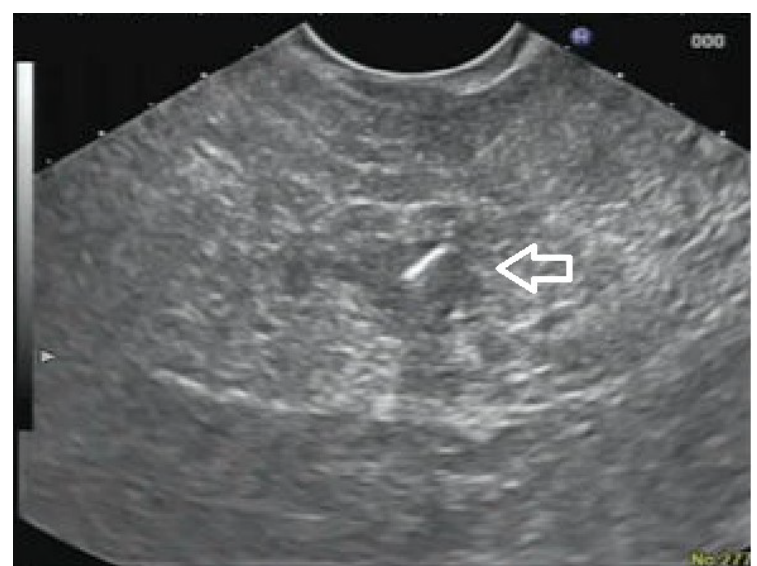

Figure 2. Endoscopic ultrasound guided placement of fiducial (white arrow).

\section{Procedure}

Access: Left upper abdominal quadrant Veress technique. Five mm port placed at the site of the Veress entry and $5 \mathrm{~mm} 30$ degree angled laparoscope used to inspect the abdomen.

Ports: The patient was placed in steep reverse Trendelenburg position with left side slightly higher. Two $5 \mathrm{~mm}$ ports were placed in the right upper abdominal quadrant, and one $5 \mathrm{~mm}$ port was placed in the midline below the xiphoid process. A $10 \mathrm{~mm}$ port was placed in the midline above the umbilicus.

Exposure: A $5 \mathrm{~mm}$ flexible liver retractor was placed through the infra-xiphoid port which maintained the gallbladder and right lobe of the liver retracted cephalad. The transverse colon was reflected caudad and the duodenum was carefully reflected laterally. The lower border of the pancreas was identified and the entry point of the superior mesenteric vein (SMV) was seen at the level of the pancreatic neck and the uncinate process inferior-laterally.

Localization: The laparoscopic probe for the ultrasound was used to localize this small deep tumor in the uncinate. The fiducials were clearly visible even before the tumor was appreciated in its entirety.

Enucleation: Using the "L" hook on the Bovie ${ }^{\oplus}$ approximately 3 $\mathrm{mm}$ of superficial pancreatic tissue was dissected through to reach the tumor. Repeat visualization of the fiducial guided the dissection. Vascular and ductal structures were avoided with the aid of intraoperative ultrasound. The tumor was well circumscribed and was "shelled out" in its entirety following its natural cleavage plane. The specimen was extracted in an Endo Catch ${ }^{\mathrm{mw}}$ (Covidien) bag through the $10 \mathrm{~mm}$ port.

Confirmation: We confirmed the presence of the fiducials and tumor extra-corporeally with the ultrasound over the specimen. We confirmed the presence of the fiducials by gross pathology (Figure 3) and the complete resection of the neuroendocrine tumor with negative margins on frozen section.

Closure: The resection area was plugged with a tongue of vascularized omentum which was secured with an absorbable suture. A 19 French BLAKE $^{\otimes}$ silicone drain (Ethicon) was placed in the resection field. The end of the drain was brought out the most lateral $5 \mathrm{~mm}$ port. The fascial defect of the $10 \mathrm{~mm}$ port was closed. The operative time for the entire procedure including intra-operative pathologic evaluation was approximately 240 minutes with an estimated blood loss of less than $50 \mathrm{ml}$. 


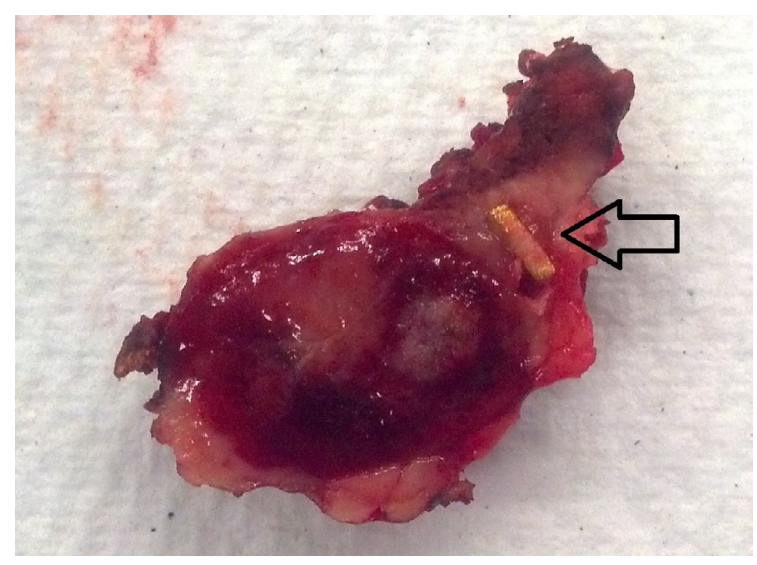

Figure 3. Gross pathology of tumor and fiducial (black arrow)

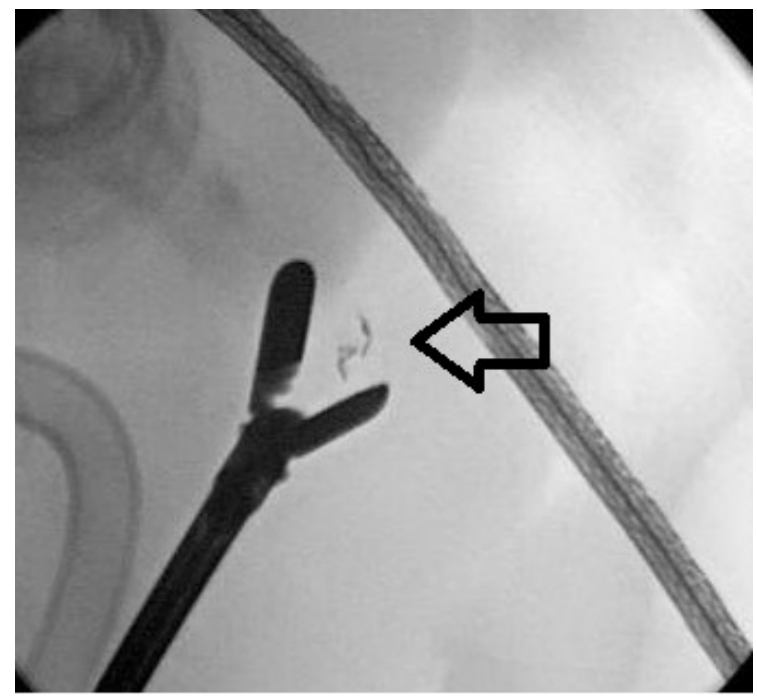

Figure 4. Intraoperative fluoroscopy identifying the fiducial (black arrow).

\section{Clinical course}

The aforementioned patient progressed without incident until postoperative day 6 when he developed a fever and abdominal pain. A computed tomography identified a $7 \mathrm{~cm}$ fluid collection, which was drained and broad spectrum antibiotics were started along with ocreotide. An endoscopic retrograde cholagiopancreatography (ERCP) revealed a fistula for which a stent was placed in the ventral duct. The patient was subsequently discharged home with a low-output fistula ( $<150 \mathrm{cc} /$ day) on postoperative day 11. One month later, drain output decreased to a scant amount. Repeat ERCP revealed resolution of the fistula, and the drain was removed. At one and two year follow up the patient is without sequela of disease or complication, and magnetic resonance imaging without evidence of residual or recurrent tumor.

Patient B. Fifty-four year old female with vague abdominal pain was found to have a $4 \mathrm{~mm}$ PNET in the tail on axial imaging. She underwent a laparoscopic distal pancreatectomy. The tumor was not able to be visualized with intraoperative ultrasound but was easily identified with intraoperative fluoroscopy. The precise localization of this tumor allowed for parenchymal preservation via a limited resection of just the portion of the pancreatic tail with splenic preservation where the tumor was located. On final pathology the tumor was only $2.5 \mathrm{~mm}$ in maximal dimension. The patient had an uneventful recovery and was discharged on the sixth postoperative day. There were no postoperative indicators of pancreatic insufficiency.

Patient C. Seventy-two year old female with multiple comorbidities (morbid obesity, DM, HTN, CKD, PE) and a $16 \mathrm{~mm}$ symptomatic insulinoma in the mid-body of the pancreas based on a CT scan. Intraoperative ultrasound and fluoroscopy identified the tumor more distal than previously thought, again allowing for greater parenchymal preservation via limited distal pancreatectomy with splenic preservation. The tumor was $22 \mathrm{~mm}$ on final pathology. The patient had an uneventful recovery and was discharged on the eighth postoperative day. There were no postoperative indicators of pancreatic insufficiency.

\section{Discussion}

This is the first case series which reports the minimally invasive resection of pancreatic neuroendocrine tumors facilitated by preoperative endoscopic ultrasound placement of fiducials. Laparoscopic enucleation of pancreatic tumors has been performed for over 20 years [41,42]. More recently this approach has become quite popular for PNETs [24-30, 43,44]. While there have been reports of laparoscopic resection of the entire uncinate process for relatively larger tumors these are still quite uncommon [45-47]. Two factors continue to limit the widespread application of laparoscopic enucleation of PNETs of the pancreatic head and uncinate. The first factor is difficulty in localizing small and deep tumors intra-operatively. We believe that preoperatively EUS-placed fiducials will facilitate intraoperative localization using ultrasound and/or fluoroscopy (Figure 4) and guide a parenchymal persevering resection based on our growing experience and the report of its use in open enucleation of PNETs of the uncinate [40]. The second factor is the high pancreatic fistula rate. It has been stated with regard to laparoscopic enucleation that "laparoscopy seems to be of no use in right-sided procedures. Pancreatic fistula is still the main cause of long-lasting morbidity"[48]. Factors associated with increased pancreatic fistula development after enucleation include tumor depth (73\% in depths $>3 \mathrm{~mm} v$ s. $30 \%$ for superficial tumors) and tumor proximity to the main pancreatic duct $(60 \% \leq 2 \mathrm{~mm}$ versus $19 \%>2 \mathrm{~mm}$ ) $[49,50]$.

In the future we hope there will be enough cases to objectively validate this proof-of-concept report. Until then, the clinical benefits and cost effectiveness of this technique are anecdotal.

\section{Conclusions}

We demonstrate that the pre-operative placement of EUS-guided fiducials facilitates the successful minimally invasive (laparoscopic and/or robotic) parenchymal preserving (enucleation and/or limited distal pancreatectomy) of a small tumors of the pancreas. High volume pancreatic centers, gastroenterologists performing advanced endoscopic procedures, and pancreatic surgeons with an advanced minimally invasive surgical skill set will benefit most from this specialized approach.

\section{References}

1. Sharma SS, Maharshi S (2008) Two stage endoscopic approach for management of choledocholithiasis during pregnancy. J Gastrointestin Liver Dis 17: 183-185. [Crossref]

2. Al-Hashem H, Muralidharan V, Cohen H, Jamidar P (2009) Biliary disease in pregnancy with an emphasis on the role of ERCP. J Clin Gastroenterol 43: 58-62. [Crossref]

3. Fine S, Beirne J, Delgi-Esposti S, Habr F (2014) Continued evidence for safety of endoscopic retrograde cholangiopancreatography during pregnancy. World $J$ Gastrointest Endosc 6: 352-358. [Crossref] 
4. Smith I, Gaidhane M, Goode A, Kahaleh M (2013) Safety of endoscopic retrograde cholangiopancreatography in pregnancy: Fluoroscopy time and fetal exposure, does it matter? World J Gastrointest Endosc 5: 148-153. [Crossref]

5. Leo M Di, Arcidiacono P (2013) Fetal radiation exposure: Is monitoring really needed? World J Gastrointest Endosc 5: 366-368. [Crossref]

6. Stimac D, Stimac T (2011) Acute pancreatitis during pregnancy. Eur J Gastroentero Hepatol 23: 839-844. [Crossref]

7. Agcaoglu O, Ozcinar B, Gok A, Yanar F, Yanar H, Ertekin C, et al. (2013) ERCP without radiation during pregnancy in the minimal invasive world. Arch Gynecol Obstet 288: 1275-1278. [Crossref]

8. Polydorou A, Karapanos K, Vezakis A, Melemeni A, Koutoulidis V, Polymeneas G, et al. (2012) A multimodal approach to acute biliary pancreatitis during pregnancy: A case series. Surg Laparosc Endosc Percutan Tech 22: 429-432. [Crossref]

9. ASGE, Maple JT, Ben-Menachem T, Anderson MA, Appalaneni V, et al. (2010) The role of endoscopy in the evaluation of suspected choledocholithiasis. Gastrointest Endosc 71: 1-9.
10. Furukawa T, Oohashi K, Yamao K, Naitoh Y, Hirooka Y, Taki T, et al. (1997) Intraductal ultrasonography of the pancrease: Development and clinical potential. Endoscopy 29 561-569. [Crossref]

11. Wu W, Faigel D, Sun G, Yang Y (2014) Non-radiation endoscopic retrograde cholangiopancreatography in the management of choledocholithiasis during pregnancy. Dig Endosc 26: 691-700. [Crossref]

12. Vohra S, Holt E, Bhat Y, Kane S, Shah J, Binmoeller K (2014) Successful singlesession endosonography-based endoscopic retrograde cholangiopancreatography without fluoroscopy in pregnant patients with suspected choledocholithiasis: A case series. J Hepatobiliary Pancreat Sci 21: 93-97. [Crossref]

13. Shaffer EA (2006) Epidemiology of gallbladder stone disease. Best Pract Res Clin Gastroenterol 20: 981-996. [Crossref]

14. Driedel D, Stavropoulos S, Iqbal S, Cappell M (2014) Gastrointestinal endoscopy in the pregnant woman. World J Gastrointest Endosc 6: 256-267. [Crossref]

Copyright: $(02016$ Mallipeddi MK. This is an open-access article distributed under the terms of the Creative Commons Attribution License, which permits unrestricted use, distribution, and reproduction in any medium, provided the original author and source are credited. 